\title{
Erratum to: Physiological employment standards II: developing and implementing physical employment standards for safety-related occupations
}

\author{
V. Jamnik • R. Gumienak $\cdot$ N. Gledhill
}

Published online: 15 June 2013

(C) Springer-Verlag Berlin Heidelberg 2013

Erratum to: Eur J Appl Physiol

DOI 10.1007/s00421-013-2603-1

The article title should read "Physiological employment standards II: developing and implementing physical employment standards for safety-related occupations."

The online version of the original article can be found under doi:10.1007/s00421-013-2603-1.

V. Jamnik $(\bowtie)$

Faculty of Health, School of Kinesiology and Health Science,

York University, Room 355 Norman Bethune College,

4700 Keele Street, Toronto, ON M3J 1P3, Canada

e-mail: ronij@yorku.ca

R. Gumienak

Faculty of Health, School of Kinesiology and Health Science,

York University, Room 358 Norman Bethune College,

4700 Keele Street, Toronto, ON M3J 1P3, Canada

e-mail: robbie5@yorku.ca

\section{N. Gledhill}

Faculty of Health, School of Kinesiology and Health Science,

York University, Room 356 Norman Bethune College,

4700 Keele Street, Toronto, ON M3J 1P3, Canada

e-mail: ngledhil@yorku.ca 